\title{
Caractérisations mécaniques des charnières pour meuble
}

\author{
Mohamad Ali*, Mohamed El-Gohary \\ Beirut Arab University, Department of Mechanical Engineering, P.O.Box 11 - 50 - 20 Riad El Solh \\ 11072809 - Beirut, Lebanon
}

\begin{abstract}
Résumé. Le travail présenté est destiné pour étudier le comportement mécanique de la liaison porte-caisson à travers des charnières invisible à encastrer pour assurer la rotation de la porte durant son ouverture et fermeture. Dans le monde de l'industrie du meuble on confronte toujours la fabrication des portes des placards dont il faut sélectionner le type et le nombre des charnières. L'étude consiste à réaliser une analyse analytique sur un cas spécifique dont on a utilisé des panneaux en fibres à densité moyenne ou MDF (medium density fiber board) et des charnières invisibles à encastrer cette étude a été suivie par une étude numérique avec un modèle des éléments finis.
\end{abstract}

Mots clés : Charnière invisible, panneaux MDF, Analyse des éléments finis, assemblage porte caisson.

\section{Introduction}

La charnière utilisée pour assurer la rotation de la porte d'un placard en bois a connue beaucoup de développement dont on a passé d'une charnière simple visible de l'extérieur à une charnière invisible. Avant quelques dizaines des années on fabriquait des meubles en bois massif qui tient avec le temps, le coût du bois massif a monté énormément. La disponibilité des machines et des processus pour fabriquer des panneaux MDF à offrir une matière première moins chère et disponible. Ces panneaux sont constitués de fibres de bois et d'un liant synthétique à base de résine que l'on soumet à des contraintes de températures et de pression $[1,2]$. Les études antérieures dans ce domaine sont concentrées dans leurs plupart sur les éléments de fixations utilisés pour assembler les différentes pièces des meubles et notamment l'utilisation des vis et leurs interactions avec les panneaux MDF [3]. Le comportement mécanique et la configuration des charnières dans l'industrie des meubles ont été étudiés tout en utilisant des outils numériques comme l'analyse par éléments finis [4]. Nous allons travailler sur un assemblage d'une porte d'un placard en bois sur un caisson en utilisant une charnière invisible à encastrer (Fig. 1). La fixation de la charnière sur la porte d'un côté et sur le caisson de l'autre côté est assurée par l'utilisation des vis coniques utilisés spécifiquement pour assembler des pièces en bois (Fig. 2). L'étude consiste à réaliser un modèle numérique dont ses résultats vont être comparer à ceux obtenus analytiquement.

\footnotetext{
* Corresponding author: mohamad.ali@bau.edu.lb
} 


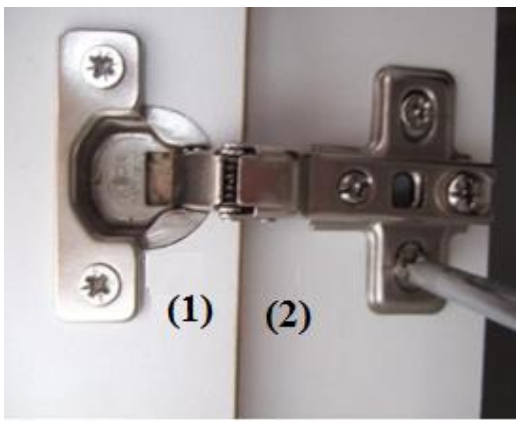

Fig. 1. Montage de la charnière, (1) côté porte et (2) coté caisson.

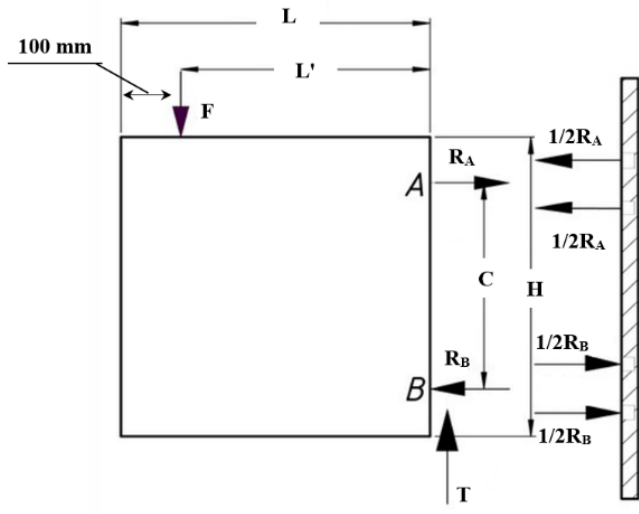

Fig. 2. Les paramètres géométriques et les réactions dans un assemblage avec des charnières.

\section{Etude analytique et numérique}

La détermination de la contrainte dans le bois du caisson autour de la vis est basée sur l'effet qu'on va prendre en compte lorsqu'on tire axialement la vis pour le faire sortir de son alésage. Deux effets sont prises en compte, l'effet du cisaillement et l'effet de la compression. L'étude numérique concerne à créer un modèle numérique en utilisant le programme Abaqus/CAE version V6-13. Ce modèle consiste à créer les différentes pièces à utiliser : une porte, un caisson et deux charnières invisible à encastrer. Les matériaux des pièces sont : Bois pour la porte et le caisson $(E=2,75 \mathrm{GPa}, v=0,28)$. Acier pour les charnières $(\mathrm{E}=200 \mathrm{GPa}$, $v=0,3)$. Le chargement est assuré par un effort concentré sur la porte avec une magnitude de $300 \mathrm{~N}$. la position et la magnitude de l'effort sont choisis selon le norme européen EN 14074. Les éléments utilisés sont des éléments 3D de type C3D3R. La modélisation de la connexion entre les vis et les alésages dans les pièces (porte, caisson) présente la clé du modèle dont il faut choisir un comportement spécifique pour une zone du bois autour de la vis.

$$
\begin{gathered}
\tau=\frac{R_{A}}{2 \pi D l}=\frac{F L^{\prime}}{2 \pi C D l} \\
\sigma=\frac{R_{A}}{2 n\left(\frac{\pi D^{2}}{4}-\frac{\pi d^{2}}{4}\right)}=\frac{2 F L^{\prime}}{\pi C n\left(D^{2}-d^{2}\right)}
\end{gathered}
$$

\section{Résultats et discussions}

Une comparaison entre les valeurs des contraintes maxi. obtenues analytiquement et numériquement a été faite avec deux diamètres différentes pour les vis et trois distances entre les charnières (Fig.3). Cette comparaison montre la contrainte équivalente de Von-Mises numérique (Fig.4) est plus grande que celle analytique avec une différence maxi. qui peut aller jusqu'à 19,8\%. Ce résultat valide le modèle numérique ce qui ouvre la voie à l'exploiter 
avec toutes les configurations. L'exploitation du modèle numérique peut conduire à créer une base de données en service de l'industrie des meubles pour aider à assembler des portes des placards avec les nombres et les espaces des charnières convenables.

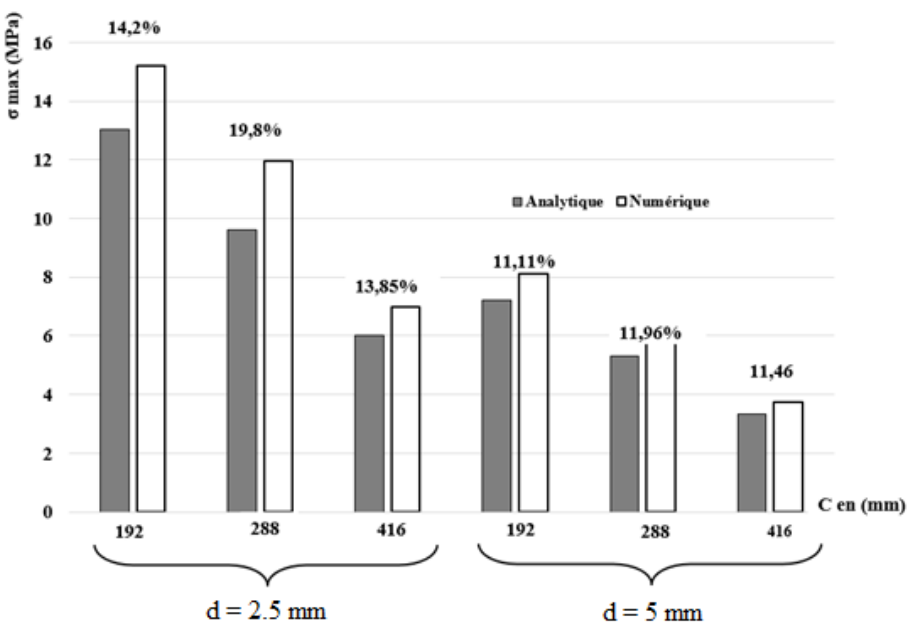

Fig.3. Les contraintes maxi des Von-Mises avec deux vis $(\mathrm{d}=2,5 \mathrm{~mm}$ et $5 \mathrm{~mm})$.

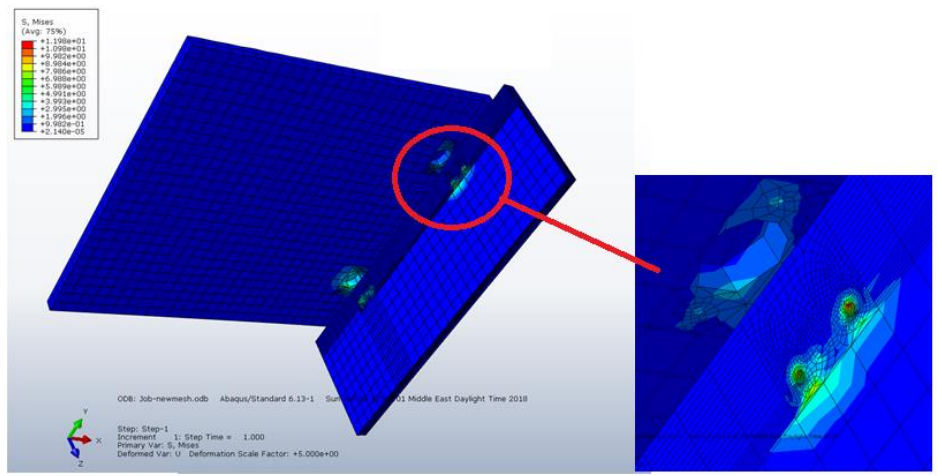

Fig.4. La contrainte de Von-Mises de la porte et le caisson

\section{Références}

1. H.Thoemen, M. Irle, M. Sernek, Wood based panels, An introduction for specialists, London, England: Brunel university press, 2010.

2. Z. Cai, R. J. Ross, Wood handbook, chapter 12 : Mechanical Properties of Wood-Based Composite Materials, General Technical Report (GTR), U.S. Dept. Of agriculture: Maidison, 2010.

3. C. A. Eckelman, "Fasteners and their use in particleboard and medium density fiberboard," National Particleboard Association, West Lafayette, Purdue University Press, 1990.

4. J. Zhou, C. Hu, S. Hu, H. Yun, G. Jiang, " Optimization of hinge configuration of furniture doors using finite elements analysis," Bioresources, vol. 7, p. $5809-5816$, 2012. 